\title{
PIC CODE SIMULATIONS OF COLLECTIVE EFFECTS IN THE SPACE- CHARGE-DOMINATED BEAM OF THE UNIVERSITY OF MARYLAND ELECTRON RING (UMER)
}

\author{
R. A. Kishek ${ }^{\#}$, S. Bernal, Y. Li, M. Reiser, and M. Venturini, \\ University of Maryland, College Park, MD \\ I. Haber, Naval Research Laboratory, Washington, DC \\ T. F. Godlove, FM Technologies, Fairfax, VA
}

Abstract.

Numerical simulation using particle-in-cell codes is a powerful tool in understanding the nonlinear dynamics of space-charge-dominated beams. The University of Maryland Electron Ring (UMER) will explore the transport of beams with intensity previously inaccessible to circular machines. The ring will also function as a testbed for accelerator codes. Applications such as heavy ion fusion and colliders require the preservation of beam quality during transport over large distances. This need for low beam emittance and small particle losses constrains the design and fabrication of the lattice and the injector. Furthermore, the non-zero energy spread leads to dispersion in the circular lattice. Simulations using the WARP code address these issues: the magnets, including the fringe field nonlinearities, are modeled realistically; dispersion matching is attempted; and effects of lattice and beam errors are examined. The simulations aid in understanding experimental results, such as the transverse density waves observed in the injector.

\section{INTRODUCTION}

Many applications of accelerators are emerging that require high intensities and good beam quality. Heavy ion inertial fusion, for example, requires transporting and accelerating a high current beam and focusing it onto a tiny spot. Spallation neutron sources and high intensity colliders also require good beam quality, although the intensities needed may be somewhat lower. Furthermore, most of these applications involve some bending of the beam, and some can benefit from the concept of a recirculator to save space and costs. The University of Maryland Electron Ring (UMER) [1], which is currently in its early construction stages, is a scaled experiment designed to investigate the physics of space-charge dominated beams in a circular geometry. A key advantage of the project is its low cost, through the use innovative features such as printed-circuit magnets [2] and modularity in design.

With a nominal $100 \mathrm{~mA}$ at $10 \mathrm{keV}$ and $50 \mathrm{~mm}-\mathrm{mrad}$ emittance, the UMER beam operates with a tune depression $\left(\mathrm{v} / \mathrm{v}_{\mathrm{o}}\right)$ of 0.14 , placing it in an extremely space-charge-dominated regime, especially for a circular machine. The intensity of the beam and the circular geometry combine to generate complications such as resonances and dispersion. Mismatches and some lattice and alignment errors play a bigger role as the beam intensity increases, especially since the compact printedcircuit magnets introduce large fringe fields that may degrade the beam quality. Image forces play a role that cannot be neglected, especially since the beam fills a large cross-section of the pipe. Finally, bunch-end effects and longitudinal confinement of the intense beam in the ring is yet another complication.

Self-consistent computer simulations are necessary because of the nonlinear nature of these effects. In the ongoing process leading up to the commissioning of the ring, the numerical simulations using the WARP code [3] are benchmarked against any experimental measurements available, some of which may be unexpected. For example, simulation has been a successful tool in understanding the radial density waves seen in a prototype injector experiment [4]. Because of its low cost and versatility, UMER will provide a valuable testbed for computer codes to be used in designing larger machines.

Since the simulation work permeates all aspects of the ring design, we have only enough space to briefly address a few of the issues listed above, directing the reader to other publications for more involved discussions.

\section{BACKGROUND}

The nominal beam current is $100 \mathrm{~mA}$ at $10 \mathrm{keV}$, resulting in a generalized perveance of 0.0015 . A nominal (unnormalized $4 * \mathrm{rms}$ ) emittance of $50 \mathrm{~mm}-\mathrm{mrad}$ and nominal average beam radius of $10.2 \mathrm{~mm}$ (for $\sigma_{\mathrm{o}}=76^{\circ}$, $\left.v_{0}=7.6\right)$ results in a tune depression $\left(v / v_{0}\right)$ of 0.14 . A future phase is planned where the beam is to be accelerated to $50 \mathrm{keV}$. Moreover, the ring is designed to run at lower beam currents, allowing us to explore a wide range of tunes.

Figure 1 displays a schematic of the ring lattice, which consists of 36 FODO cells around the $11.52 \mathrm{~m}$ circumference ring. Each cell is therefore $32.0 \mathrm{~cm}$ long and contains two evenly-spaced printed-circuit quadrupoles [2] and, in between those, a printed-circuit

\footnotetext{
* http://www.ipr.umd.edu/ ebte/ring

\# ramiak@ebte.umd.edu
} 
dipole which bends the beam by $10^{\circ}$. The quadrupole gradient is about $0.078 \mathrm{Tesla} / \mathrm{m}$, while the bending dipole peak field is about 0.00154 Tesla. Three induction gaps used for longitudinal confinement are evenly distributed around the ring, while the remainder of the spaces are occupied by diagnostics and pumping ports.

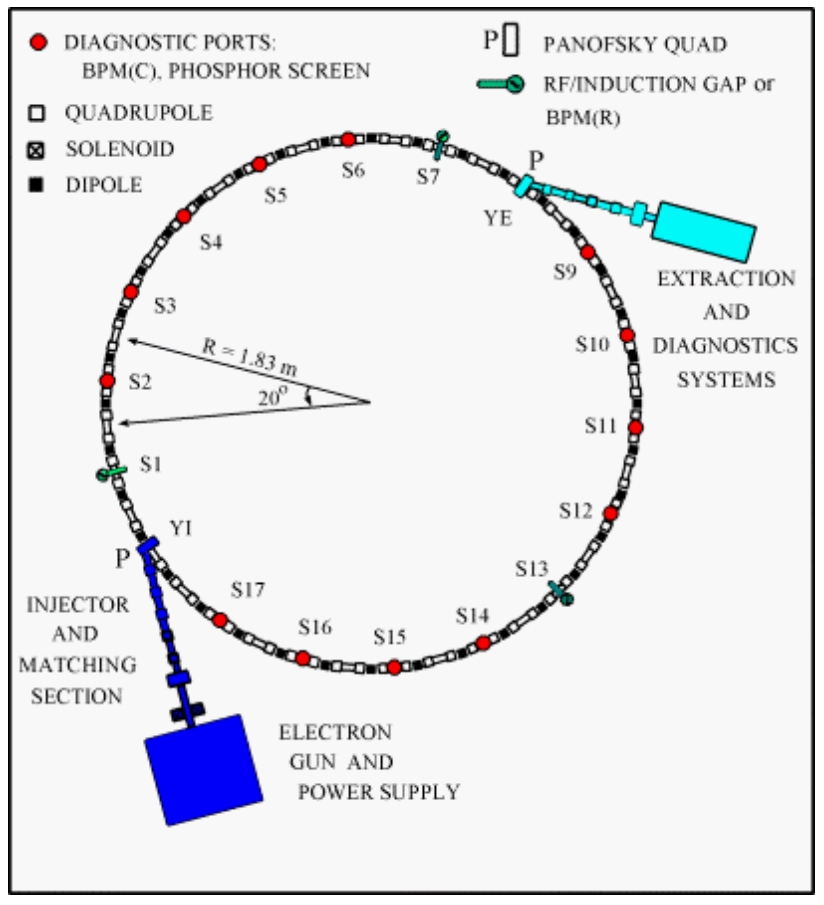

Figure 1. Schematic of ring design [1].

In the simulations described herein, we rely primarily on the WARP particle-in-cell (PIC) code [3], which has been developed at Lawrence Livermore National Laboratory (LLNL) for heavy ion fusion applications. An important feature of the WARP code is its ability to efficiently track a space-charge-dominated beam along bends. The particle orbits are integrated self-consistently under the applied fields and the self-fields. The simulations have the following characteristics: (a) the magnet nonlinearities, including those arising from the fringe fields, are fully included; (b) the numerics have been checked for convergence; and (c) although most simulations were performed using the $2 \mathrm{~d} 3 \mathrm{v}$ "slice model", certain key simulation were tested using the full $3 \mathrm{~d}$ code. Ref. [5] contains further details.

\section{SIMULATION RESULTS AND DISCUSSION}

\subsection{Magnet nonlinearities}

Despite large fringe fields, the printed-circuit magnets were designed to be linear when integrated parallel to the magnet axis. Simulations with the quadrupoles that include the fringe fields exhibit no emittance growth, confirming the designed linearity of the integrated fields [5]. Including the fringe fields of the bending dipoles, however, results in some non-numerical emittance growth (about $30 \%$ in 10 turns or 360 dipoles). The question arises as to why the dipoles, which were also designed to be linear in the integrated sense, lead to more emittance growth than the quadrupoles. The difference is that the individual particles in the beam follow a curved trajectory inside the dipole, whereas the dipole fields were designed to be linear when integrated along $\mathrm{z}$, the dipole axis. Extensive simulations [partially discussed in ref. 5] link the observed growth in emittance to the field inhomogeneities seen by the beam over its curved trajectory.

Although the emittance growth is relatively small, it can become significant if we wish to operate for $>25$ turns. A remaining question currently being explored is therefore whether a dipole redesign can alleviate the longterm emittance growth by minimizing the nonlinearities integrated over the self-consistent particle orbits.

\subsection{Dispersion Matching}

Since a beam, in general, has a non-zero energy spread, propagation in bends leads to dispersion, i.e., particles at different energies oscillate around different reference trajectories. If not explicitly matched for dispersion, the beam experiences a dispersion mismatch as it enters the ring from the straight injector, or as it leaves it to the extraction section. For a space charge dominated beam, this dispersion mismatch leads to emittance growth, as confirmed earlier by theory and simulation [5-6]. It is possible to use the generalized envelope equations developed by Venturini and Reiser [6] to design a matching section that matches both the envelopes and the dispersion function for a space-charge-dominated beam. There remain questions, however, about the applicability of the theory as the beam attempts to relax to a state of equilibrium.

We applied numerical simulations with the WARP code to evaluate the results of employing dispersion matching at both injection and extraction. The results are discussed in ref. [7] and generally indicate that proper dispersion matching can help reduce the emittance growth. Indeed, the simulations indicate that a significant component of the emittance growth from a dispersion mismatch is reversible, meaning that it can be recovered by proper matching at extraction. However, dispersion matching using the generalized envelope equations becomes more difficult at higher currents because relaxation processes develop earlier downstream, and henceforth the predictions of these equations depart from the simulation results.

\subsection{Errors, Mismatches and Misalignments}

Table 1 is a summary of the error analyses conducted so far. The dipole nonlinearities have been included in the 
error studies. By themselves, the nonlinearities contribute $31 \%$ emittance growth over 10 turns. It is clear that reasonable errors in injection or mispowering do not affect the emittance growth beyond that. These studies show that as long as the beam centroid remains confined within $1 \mathrm{~mm}$ of the pipe axis, there is negligible change in the emittance growth.

A mismatched beam experiences an additional emittance growth as the free energy in the beam is converted to thermal energy. However, after 10-15 turns the emittance growth due to the mismatch diminishes as the mismatch oscillations damp out and the beam reaches a new equilibrium. A glance at Table 1 reveals that the beam is very sensitive to errors from quadrupole rotations (skew quads), thus imposing tight tolerances on the rotation angles. This subject is treated in more detail in ref. [8].

Table 1: Summary of multi-particle error studies.

\begin{tabular}{|c|c|c|c|c|}
\hline Description & \begin{tabular}{|c|} 
Error \\
Magnitude
\end{tabular} & $\begin{array}{c}\Delta \varepsilon / \varepsilon \\
\text { (in } \mathbf{1 0} \text { Turns }\end{array}$ & $\begin{array}{c}\text { Centroid } \\
\text { Offset }(\mathbf{m m})\end{array}$ & $\begin{array}{c}\text { Particle } \\
\text { Losses }\end{array}$ \\
\hline $\begin{array}{l}\text { No Errors } \\
\text { Linear } \\
\text { Magnet Nonlinearities }\end{array}$ & Fringe Fields & $\begin{array}{r}8 \% \\
31 \%\end{array}$ & $\begin{array}{c}0 \\
0.2 \mathrm{~mm}\end{array}$ & $\begin{array}{l}0 \\
0\end{array}$ \\
\hline $\begin{array}{l}\text { Mismatches } \\
\quad \mathrm{x} \\
\mathrm{x} \text { and }-\mathrm{y}\end{array}$ & $\begin{array}{l}0.5 \mathrm{~mm} \\
0.5 \mathrm{~mm}\end{array}$ & $\begin{array}{c}41 \% \\
46 \% \\
(84 \% / 20 \mathrm{~T})\end{array}$ & & $\begin{array}{c}0 \\
0.1 \%\end{array}$ \\
\hline $\begin{array}{l}\text { Injection Errors } \\
\text { within } 1 \mathrm{~mm} \text { or } 4 \mathrm{mrad} \\
2 \mathrm{~mm} \text { or } 8 \mathrm{mrad}\end{array}$ & & $\begin{array}{l}33.5 \% \\
45 \%\end{array}$ & $\begin{array}{l}1 \mathrm{~mm} \\
2 \mathrm{~mm}\end{array}$ & $\begin{array}{l}0 \\
0\end{array}$ \\
\hline $\begin{array}{l}\text { Mispowering } \\
\text { Dipoles } \\
\text { Single Magnet } \\
\text { Systematic }\end{array}$ & $\begin{array}{l}1.0 \% \\
-2.5 \% \\
-5.0 \%\end{array}$ & $\begin{array}{l}38 \% \\
27 \% \\
38 \%\end{array}$ & $\begin{array}{l}0.3 \mathrm{~mm} \\
1 \mathrm{~mm} \\
2 \mathrm{~mm}\end{array}$ & 0 \\
\hline $\begin{array}{l}\text { Quadrupoles } \\
\text { Single Magnet }\end{array}$ & $\begin{array}{l}1.0 \% \\
0.5 \% \\
0.2 \%\end{array}$ & $\begin{array}{c}42 \% \\
35.5 \% \\
35 \%\end{array}$ & & $\begin{array}{l}0 \\
0 \\
0\end{array}$ \\
\hline $\begin{array}{l}\text { Rotation } \\
\quad \text { Quadrupoles (random) }\end{array}$ & $\begin{array}{l}0.1 \mathrm{deg} \\
0.2 \mathrm{deg}\end{array}$ & $\begin{array}{l}40 \% \\
60 \%\end{array}$ & & 0 \\
\hline
\end{tabular}

\subsection{Other Issues}

We have also applied the code to other situations, which are described more fully elsewhere. The code has played a role in designing the injector [9], especially the inflector bend, and in testing the effects of the Panofsky quad used in injection [10]. The simulations have reproduced radial density waves observed in a prototype injector experiment [4], and played a major role in linking this phenomena to the initial particle distribution at the aperture.

\section{CONCLUSION}

In this paper we have touched upon the magnet nonlinearities, dispersion matching, and error analyses, among others. In all cases, the simulation is proving to be a valuable tool in exploring complex physical phenomena that are not easily tractable by analytic means. The future plan of the simulation effort is to move towards full 3D simulation in order to (a) understand the end effects and longitudinal confinement, and (b) simulate the electron gun so as to get a better model of the initial beam distribution. As experimental data becomes available in the forthcoming commissioning process of UMER, it will be interesting to compare with the WARP predictions.

\section{ACKNOWLEDGEMENTS}

We wish to thank our colleagues D. P. Grote, J. J. Barnard, A. Friedman, S. Lund, and J.G. Wang for many fruitful discussions. We also thank K. Danylevich for assistance with presentation. The code runs on computers provided by the National Energy Research Scientific Computing Center (NERSC). This research is sponsored by the U. S. Department of Energy (DOE) under contract DE-FG02-94ER40855.

\section{REFERENCES}

[1] M. Reiser, et. al., "The Maryland Electron Ring for Investigating Space-Charge Dominated Beams in a Circular FODO System," these proceedings, TUCR3.

[2] T. F. Godlove, S. Bernal, and M. Reiser, Proc. 1995 Particle Accelerator Conference, 2117 (1995); also W. W. Zhang, "Magnetic Field Measurement of Printed-Circuit Quadrupoles and Dipoles," these proceedings, THP170.

[3] D. P. Grote, et. al., Fus. Eng. \& Des. 32-33, 193-200 (1996).

[4] S. Bernal, R. A. Kishek, M. Reiser, and I. Haber, "Observations and Simulations of Transverse Density Waves in a Collimated, Space-Charge-Dominated Electron Beam," accepted in Phys. Rev. Lett (1999); also these proceedings, TUP115.

[5] R. A. Kishek, et. al., "Unique Features of the University of Maryland Electron Ring and the Necessity of PIC Code Simulation," proc. ICAPS98, Monterey, CA, in print (1999); and references therein.

[6] M. Venturini and M. Reiser, "rms Envelope Equations in the Presence of Space Charge and Dispersion," Phys. Rev. Lett., 81, 96 (1998);

[7] M. Venturini, et. al., "The problem of dispersion matching in space charge dominated beams," these proceedings, THP134.

[8] R. Kishek, et. al., "Effects of Quadrupole Rotations on the Transport of Space-Charge-Dominated Beams: Theory and simulations Comparing Linacs with Circular Machines," these proceedings, TUP119.

[9] T. Godlove, et. al., "The $10 \mathrm{keV}$ Injector for the University of Maryland Electron Ring Project," these proceedings, WEA36.

[10] Y. Li, et. al., "Design, simulation and test of Panofsky quadrupole," these proceedings, THP169. 